\title{
Transformation of the Topological Phase and the Edge Modes of Double-Bilayer Bismuthene with Inter-Bilayer Spacing
}

\author{
Huanzhi Hu ${ }^{1}$, Zhibin Shi ${ }^{1}$, Peng Wang ${ }^{2}$, Weiping Zhou ${ }^{1}$, Tai-Chang Chiang ${ }^{3,4,5}$ and \\ Xiaoxiong Wang ${ }^{1, *}$ \\ 1 Department of Applied Physics, Nanjing University of Science and Technology, Nanjing 210094, China; \\ huhuanzhi@njust.edu.cn (H.H.); szb941211@163.com (Z.S.); wpzhou@njust.edu.cn (W.Z.) \\ 2 College of Electronic, Communication and Physics, Shandong University of Science and Technology, \\ Qingdao 266590, China; phywangp@sdust.edu.cn \\ 3 Department of Physics, University of Illinois at Urbana-Champaign, 1110 West Green Street, Urbana, \\ IL 61801-3080, USA; tcchiang@illinois.edu \\ 4 Frederick Seitz Materials Research Laboratory, University of Illinois at Urbana-Champaign, 104 South \\ Goodwin Avenue, Urbana, IL 61801-2902, USA \\ 5 Department of Physics, National Taiwan University, Taipei 10617, Taiwan \\ * Correspondence: phywangxx@njust.edu.cn
}

Received: 4 April 2019; Accepted: 20 May 2019; Published: 22 May 2019

\begin{abstract}
The transformations of the topological phase and the edge modes of a double-bilayer bismuthene were investigated with first-principles calculations and Green's function as the inter-bilayer spacing increased from $0 \AA$ to $10 \AA$. At a critical spacing of $2 \AA$, a topological phase transition from a topological insulator to a band insulator resulting from a band inversion between the highest valence band and the second lowest conduction band, was observed, and this was understood based on the particular orbital characters of the band inversion involved states. The edge modes of double-bilayer bismuthene survived the phase transition. When $d$ was $2 \AA<d<4 \AA$, the interaction between the edge modes of two separated bismuthene bilayers induced an anti-crossing gap and resulted in a trivial band connection. At and beyond $4 \AA$, the two bilayers behavior decoupled entirely. The results demonstrate the transformability of the topological phase and the edge modes with the inter-bilayer spacing in double-bilayer bismuthene, which may be useful for spintronic applications.
\end{abstract}

Keywords: double-bilayer bismuthene; topological phase transition; edge modes; inter-bilayer spacing; first-principles calculations

\section{Introduction}

Topological insulators have attracted much attention over the past decade for their fundamental physics interests and promising applications in spintronics [1]. A topological insulator is different from a band insulator in regard to band topology, which can be characterized by a topological invariant [2]. The nontrivial topology of the band structure of a topological insulator gives rise to metallic boundary modes [1], which are robust and cannot be destroyed by altering the boundary conditions $[3,4]$. Moreover, the edge modes are highly spin polarized because of the prominent spin-orbit coupling effect associated with the heavy elements commonly found in topological insulators [5]. The spin polarized boundary modes can host spin currents, which can be utilized to transmit and process information with little energy dissipation [6]. Although the topological edge modes cannot be eliminated easily, their properties can be tuned by factors such as strain [7], composition [8] and chemical adsorption [9]. 
The tunability of the topological boundary modes is crucial for the applications of topological insulators in spintronics.

Bismuthene [10], a monolayer of Bi with a buckled graphene-like hexagonal lattice [11,12], has a large, nontrivial band gap. We call such a sheet of Bi atomic crystal a bilayer (BL). It is a very promising topological insulator for spintronics application above room temperature. Since it was theoretically predicted to be a two-dimensional (2D) topological insulator, it has inspired intensive research [9,13-20]. Liu et al. studied the band topology of ultrathin Bismuth films (1 4 bilayers), and found that the nontrivial nature of Bismuth films is independent of film thickness [15]. Besides film thickness, the effects of strain, substrate and chemical modification have been explored as well $[9,16-19,21]$. In contrast, the inter-bilayer spacing as an important parameter to tune the property of double-bilayer bismuthene has not been well investigated up until now, especially the effect of inter-bilayer interaction on the properties of edge mode having not yet been reported to the best of our knowledge. Liu et al. reported the topological phase transition in double-bilayer bismuthene induced by increasing inter-bilayer spacing to $2 \AA$ [15], but the band inversion mechanism was not clearly revealed. As we will present later, the band inversion detail is distinct from usual cases.

In the present work, we studied the evolution of the electronic structure of double-bilayer bismuthene from a strongly coupled double-bilayer to two decoupled bismuthene bilayers as the inter-bilayer spacing increases. The system underwent a phase transition from a topological insulator to a band insulator through a singular point of a semimetal phase; however, the band inversion involved an unusual interweaving of three, rather than just two bands. The band inversion occurred between the highest valence band and the second lowest conduction band rather than between the two bands closest to the Fermi level as usual. When the inter-bilayer spacing was between $2 \AA$ and $4 \AA$, the interaction between the edge modes of the two bilayers induced an anti-crossing gap and made the whole system topologically trivial; the trivial nature was preserved even when the spacing was beyond $4 \AA$ and the anti-crossing gap disappeared. Our findings demonstrate the transformability of the topological phase and the dispersion of edge modes with inter-bilayer spacing of double-bilayer bismuthene.

\section{Calculation Method}

The mono- and double-bilayer bismuthene systems were modeled by a supercell approximation. The vacuum gap between image slabs was $20 \AA$ and the interaction between neighboring slabs was negligible as verified by its insensitivity to the change of the vacuum gap size. The calculations were performed using the Abinit package (v8.4.3) $[22,23]$ within the local density approximation (LDA) as implemented in the Hartwigsen-Goedecker-Hutter (HGH) pseudopotential [24]. Previous results show that even LDA is good enough to reach a reliable conclusion for the present system [15]. The employment of Heyd-Scuseria-Ernzerhof (HSE) hybrid functional can certainly improve the accuracy of the prediction of band gap [20], but it does not change the conclusion of the nontrivial property of bismuthene. The cutoff of kinetic energy was $400 \mathrm{eV}$ and the integration was carried out on a $\Gamma$-centered $17 \times 17 \times 1$ grid in accordance with the Monkhorst-Pack scheme [25]. The spectra of edge modes were calculated with WannierTools package (v2.4.0) [26] based on the Green's function method.

\section{Results and Discussion}

The band structures of mono-bilayer and double-bilayer bismuthene are reproduced and shown in Figure 1a,c for reference; the corresponding atomic models are presented in Figure 1b,d. Both the mono-bilayer and double-bilayer bismuthene had a gap spanning the whole Brillouin zone. The gaps were $0.49 \mathrm{eV}$ and $0.04 \mathrm{eV}$ for mono-bilayer and double-bilayer bismuthene, respectively. A mono-bilayer bismuthene has a graphene-like honeycomb lattice with an in-plane lattice constant $4.38 \AA$ and a substantial buckling of $1.74 \AA$, belonging to the P $\overline{3} \mathrm{~m} 1$ space group (\#164). Double-bilayer bismuthene is constructed by stacking two mono-bilayers in an A-B configuration, where the top bilayer is shifted along the red arrow-as indicated in Figure 1d-relative to the bottom bilayer. Because of the inter-bilayer interaction, the buckling in double-bilayer is reduced to $1.64 \AA$ after energy 
minimization. The band topology analysis on mono-bilayer and double-bilayer indicated that both of them were topologically nontrivial. The results are well consistent with previous reports $[12,15,20]$, which suggests that the calculation settings we adopted in our present study are reliable, and we used the settings to perform the rest of the calculations.

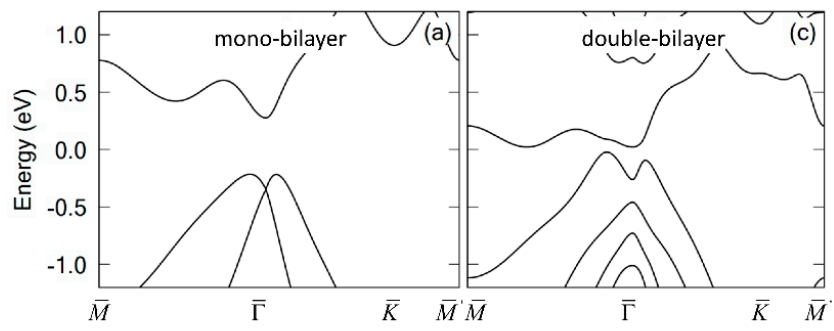

(b)
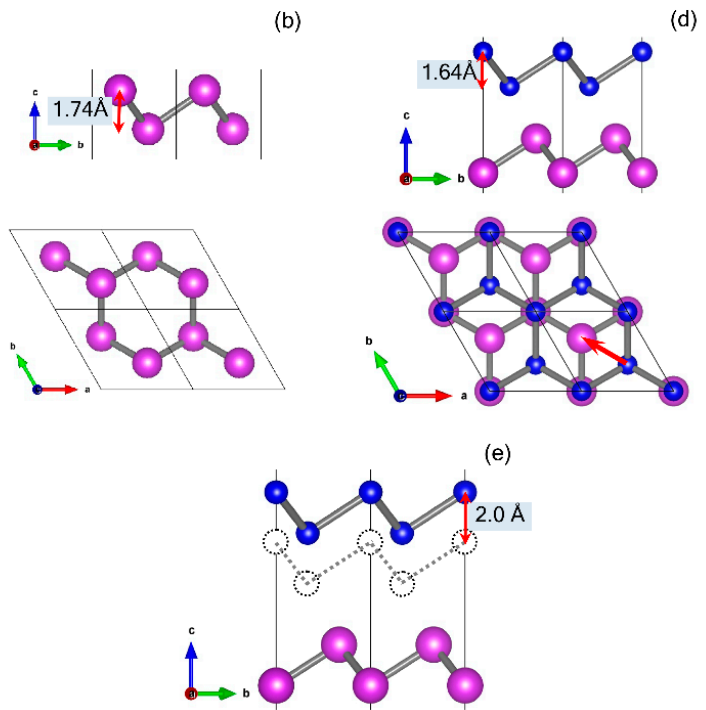

Figure 1. Band structure (a) and ball-and-stick model (b) of mono-bilayer bismuthene. The intra-bilayer buckling is indicated. (c) and (d) are the band structure and atomic model of double-bilayer bismuthene, respectively. The top bilayer Bi atoms are represented by small blue balls and the bottom bilayer by big magenta balls. The intra-bilayer buckling is indicated as well. The red arrow in (d) indicates how the two bilayers of bismuthene are stacked and shifted to form a double-bilayer bismuthene. (e) is the atomic model of double-bilayer bismuthene with $2.0 \AA$ extra inter-bilayer spacing. The position of the top bilayer in equilibrium geometry is indicated with dotted circles.

Since both mono-bilayer and double-bilayer bismuthene were topologically nontrivial, an interesting question is, how are these two cases connected? In other words, what happens when the two bilayers in double-bilayer bismuthene are separated from each other and ultimately form two decoupled mono-bilayers? To address this issue, we performed band calculations for double-bilayer bismuthene for various inter-bilayer spacings. Although Liu et al. noticed a topological phase transition when the extra inter-bilayer spacing increased to $2 \AA$ [15], the detailed band inversion mechanism was still lacking. Here, we present more thorough results and discussion about the phase transition. The results are shown in Figure 2. In the simulation, we simply increased the spacing between the two bilayers from 0 to $10 \AA$, and no structure relaxation was performed after introducing the extra spacing. The configuration of double-bilayer bismuthene with $2 \AA$ extra spacing is shown in Figure 1e. The $0 \AA$ case is included here for reference. With $1 \AA$ extra spacing (Figure 2b), band V1 moved up, band C2 moved down significantly and band C1 also moved down slightly. The gap at the zone center became much smaller. At $2 \AA$ extra spacing (Figure 2c), the gap became zero and the system transformed into a semimetal state. Interestingly, the two conduction bands, $\mathrm{C} 1$ and $\mathrm{C} 2$, and the valence band V1 became degenerate at the zone center. Bands V1 and C2 combined to form a Dirac 
cone, giving rise to massless fermions, while band $\mathrm{C} 1$ gave rise to massive fermions. Further increasing of the inter-bilayer spacing caused gap opening, but bands $\mathrm{C} 1$ and $\mathrm{C} 2$ remained degenerate at the zone center, as the downward motion of $\mathrm{C} 2$ being arrested by C1. At $10 \AA$ extra spacing, the system became essentially two isolated mono-bilayers of bismuthene (Figure 2f), where each state was doubly degenerate. The band structure was perceptibly different from that shown in Figure 1a for an isolated mono-bilayer because of the slight difference in structure; no geometrical relaxation was performed during the separation of double-bilayer bismuthene, so each bilayer was slightly different from a free standing mono-bilayer in structure. In Figure 2, the parity of some states is indicated. There was a net parity reversal across the gap as the extra spacing passed through $2 \AA$, suggesting a topological phase transition. If van der Waals (vdW) force correction [27] is included in the calculation, the topological phase transition occurs at $d=2.16 \AA$. The specific functionals adopted in the calculation may change the critical spacing of phase transition, but the transition will happen at a certain $d$ value regardless. In this sense, the phase transition is independent on the employed functionals. The insets in Figure $2 b, d$ show the real parts of the wave functions for the states as indicated by the arrows. Blue and yellow represent negative and positive values. These results further illustrate the band inversion between $\mathrm{C} 2$ and V1 when the extra spacing increases from 1 to $3 \AA$. Normally, band inversion occurs between the lowest conduction band and the highest valence band. For the present case, there was an extra "spectator" band $\mathrm{C} 1$, which entangled with $\mathrm{C} 2$. The insets revealed that band $\mathrm{C} 1$ at the zone center had a charge distribution highly localized within the Bi atomic planes, and thus it was not very sensitive to the variations in the inter-bilayer spacing. By contrast, bands C2 and V1 at the zone center had more $p_{z}$ like characteristics, and thus they were more sensitive to the variations in the inter-bilayer spacing.
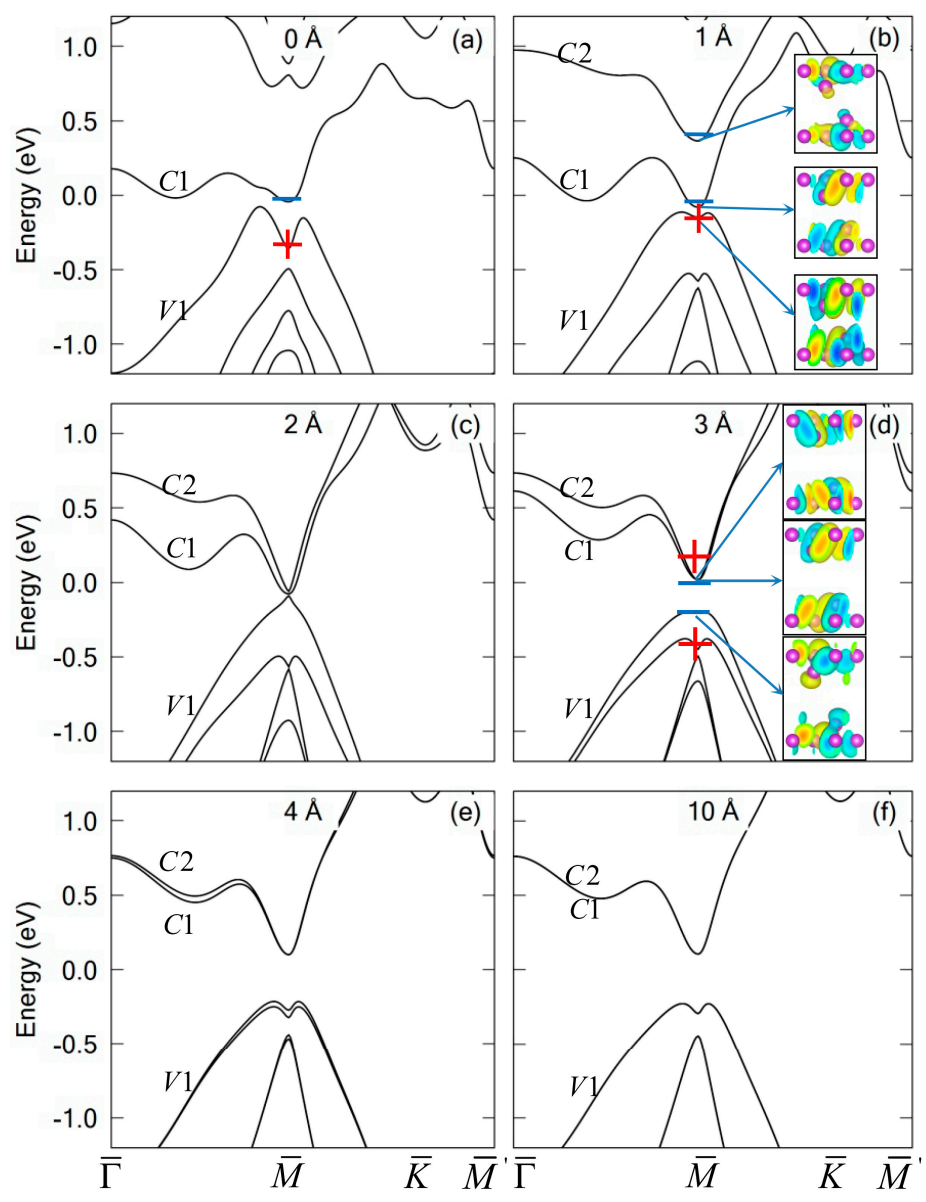

Figure 2. Band structures of double-bilayer bismuthene by scaling the inter-bilayer spacing as indicated. The parity of some states at the zone center is indicated. The insets in (b) and (d) show the real parts of the wave functions for the states at the zone center as indicated. 
Based on the analysis above, double-bilayer bismuthene, which is topologically nontrivial, goes through a topological phase transition at an extra inter-bilayer spacing of $d=2 \AA$. At $d>2 \AA$, the system should become topologically trivial, including the case where the two bilayers were well separated and decoupled. However, each mono-bilayer bismuthene by itself was topologically nontrivial. Thus, the question is, are two well-separated mono-bilayers topological or not? The answer to this question depends on the point of view. If the two bilayers are regarded as a single system (with a small but non-zero coupling), the system as a whole was topologically trivial, even though each of the bilayers by itself were topologically nontrivial. Let the parity product over all time-reversal invariant momentum (TRIM) points for each isolated bilayer be $N$ (minus one) relative to its own inversion center, and it is straightforward to show that the parity product for the two minimally coupled bilayers was $N^{2}=1$ relative to the inversion center of the double-bilayer, and thus the composite system was trivial even though each of the two components by itself were nontrivial.

To further elucidate the unusual band inversion process involving a spectator band, a tight-binding model including interactions up to the second neighbors under the Slater-Koster scheme [28] was built. The basis set is $\left|s, p_{x}, p_{y}, p_{z}\right\rangle|\uparrow \downarrow\rangle$. The Hamiltonian is a $32 \times 32$ matrix $H=\left(\begin{array}{cc}H_{1} & \delta H_{2} \\ \delta H_{2}^{*} & H_{1}\end{array}\right)$, where $H_{1}$ and $H_{2}$ are two $16 \times 16$ submatrices representing the intra-bilayer and inter-bilayer interactions, respectively. The scaling factor $\delta$ ranges from 0 to 1 . When it is set to 0 , i.e., the inter-bilayer coupling is turned off, the system corresponds to two well-separated, decoupled mono-bilayers. When $\delta$ is set to 1 , the system corresponds to a double-bilayer with its normal inter-bilayer spacing. The tight-binding parameters determined by fitting the density functional theory (DFT) bands of double-bilayer bismuthene with $2 \AA$ spacing are shown in the Table 1.

Table 1. Tight-binding parameters for double-bilayer bismuthene: $E_{S}$ and $E_{p}$ are on-site energies of the $s$ and $p$ orbitals, respectively, $\Delta_{S O C}$ is spin-orbit splitting and the other factors are Slater-Koster type integrals. $I(N N)$ and $I(S N N)$ stand for the intra-bilayer nearest neighbors and second-nearest neighbors, respectively, while $O(N N)$ and $O(S N N)$ are the inter-bilayer nearest neighbors and second-nearest neighbors, respectively. All energies are in the unit of eV.

\begin{tabular}{ccccc}
\hline & $E_{S}$ & $E_{p}$ & $\Delta_{S O C}$ & \\
& -7.8335 & -0.3665 & 1.5 & \\
& $I(N N)$ & $I(S N N)$ & $O(N N)$ & $O(S N N)$ \\
$V_{S S \sigma}$ & -0.720 & -0.086 & -0.375 & -0.225 \\
$V_{s p \sigma}$ & 1.051 & 0.126 & 0.607 & 0.376 \\
$V_{p p \sigma}$ & 1.740 & 0.209 & 0.6717 & 0.5022 \\
$V_{p p \pi}$ & -0.421 & -0.050 & -0.150 & -0.045 \\
\hline
\end{tabular}

Evolution of the band structure as a function of $\delta$ is shown in Figure 3. As the inter-bilayer coupling became weaker from Figure $3 \mathrm{a}-\mathrm{f}$, corresponding to varying $\delta$ from 1 to 0 , the conduction and valence bands around the zone center moved towards each other first. When $\delta=0.4$, the conduction and valence bands became degenerate at the zone center, where a topological phase transition occurred as expected. The linear dispersion of the Dirac cone and the six-fold degeneracy of the states were successfully reproduced by this simple tight-binding model. The detailed band shapes away from the zone center were different between the DFT and tight-binding results. This loss of accuracy can be attributed to two factors: The first is that interactions beyond the second-nearest-neighbors were not included in the model. The second is that the inter-bilayer interactions were scaled with just one coefficient $\delta$ for different bands. In reality, the interactions decayed at different rates for different states. Despite these inaccuracies, this model showed the general trend of gap closing and reopening associated with a topological phase transition as tuning the inter-bilayer spacing. The results presented here offer a foundation for further study on the evolution of edge modes of double-bilayer bismuthene ribbons as increasing spacing with a tight-binding model. 

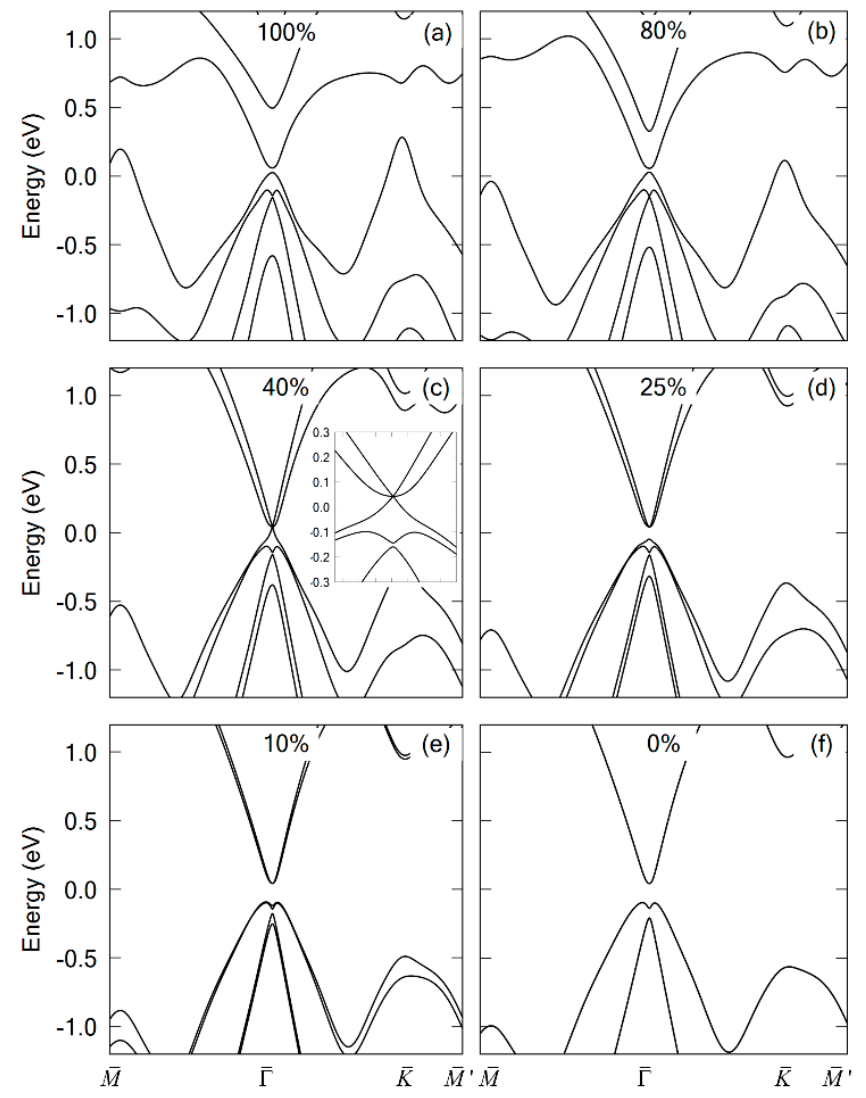

Figure 3. Band structure of double-bilayer bismuthene based on a tight binding model with the inter-bilayer hopping strength set at various levels as indicated. The inset in (c) is an enlargement of the band structure close to the Fermi level.

According to the bulk-boundary correspondence principle [1], the boundary modes of a topological phase are closely associated with the topological character of bulk. As the topological character of bulk changes with inter-bilayer spacing in double-bilayer bismuthene, the edge modes will evolve accordingly. As the properties of edge modes are critical for spintronic application, it is necessary to unveil how the dispersion of edge modes vary with inter-bilayer spacing. The spectra of the zig-zag edge along the a axis (Figure 1d) of a semi-infinite double-bilayer bismuthene with various spacings as indicated are presented in Figure 4a-f. Figure 4a shows the spectra of the edge states of a double-bilayer bismuthene at its equilibrium spacing. Because of the small band gap of double-bilayer bismuthene and the smearing of spectra, the bulk band gap at the zone center could hardly be recognized. There were three pairs of bands, indicated as B (bottom), M (middle) and T (top) (Figure 4d) in the bulk gap around $\bar{X}$. Only the $M$ pair was topologically related, which bridged the valence and conduction bands; the other two pairs, $\mathrm{T}$ and $\mathrm{B}$, were trivial. As far as spintronic applications are concerned, the presence of trivial edge modes is undesirable; this will reduce the overall spin polarization of edge modes. All of the three pairs of bands were degenerate and formed Dirac cones at the zone boundary $\bar{X}$. When the edge modes dispersed from $\bar{X}$ to $\bar{\Gamma}$, the T pair converged with the conduction bands and the $B$ pair merged with the valence bands. This observation became much clearer when the inter-bilayer spacing increased to $2 \AA$. As the spacing increased from $0 \AA$ to $3 \AA$, the system went through a topological phase transition. All of the three pairs of edge modes survived the phase transition, but the connection of the $M$ pair changed with the variation in the topological character of bulk. The most prominent change in the band of edge state occurred at $d=3 \AA$, where the system was topologically trivial, composed of two slightly coupled bismuthene bilayers. The lower branch of the $\mathrm{T}$ pair became more isolated from the bulk band continuum around the zone center, and there was a tiny anti-crossing band gap arising from the coupling between the edge modes of the two bilayers, indicated by the red arrows in 
Figure $4 \mathrm{~d}$. The gap made the lower branch of the $\mathrm{T}$ pair connect to the conduction band rather than to the valence band, which manifested the trivial nature of the system. The anti-crossing band gap aroused by the interaction between surface states has been well studied before for three-dimensional (3D) systems [29-31], but it is seldom addressed for 2D materials. Meanwhile, a Dirac cone at the zone center formed by the $\mathrm{M}$ pair became more evident with the growth of the bulk band gap size. As the inter-bilayer spacing grew to $4 \AA$, the anti-crossing gap became indistinguishable, implying the interaction between the two edge modes was negligible at this distance. This conclusion was supported by the similarity of the edge mode spectra in Figure 4e,f. The closing of the anti-crossing gap at $4 \AA$ was also verified with a simulation, including vdW and HSE06 correction.

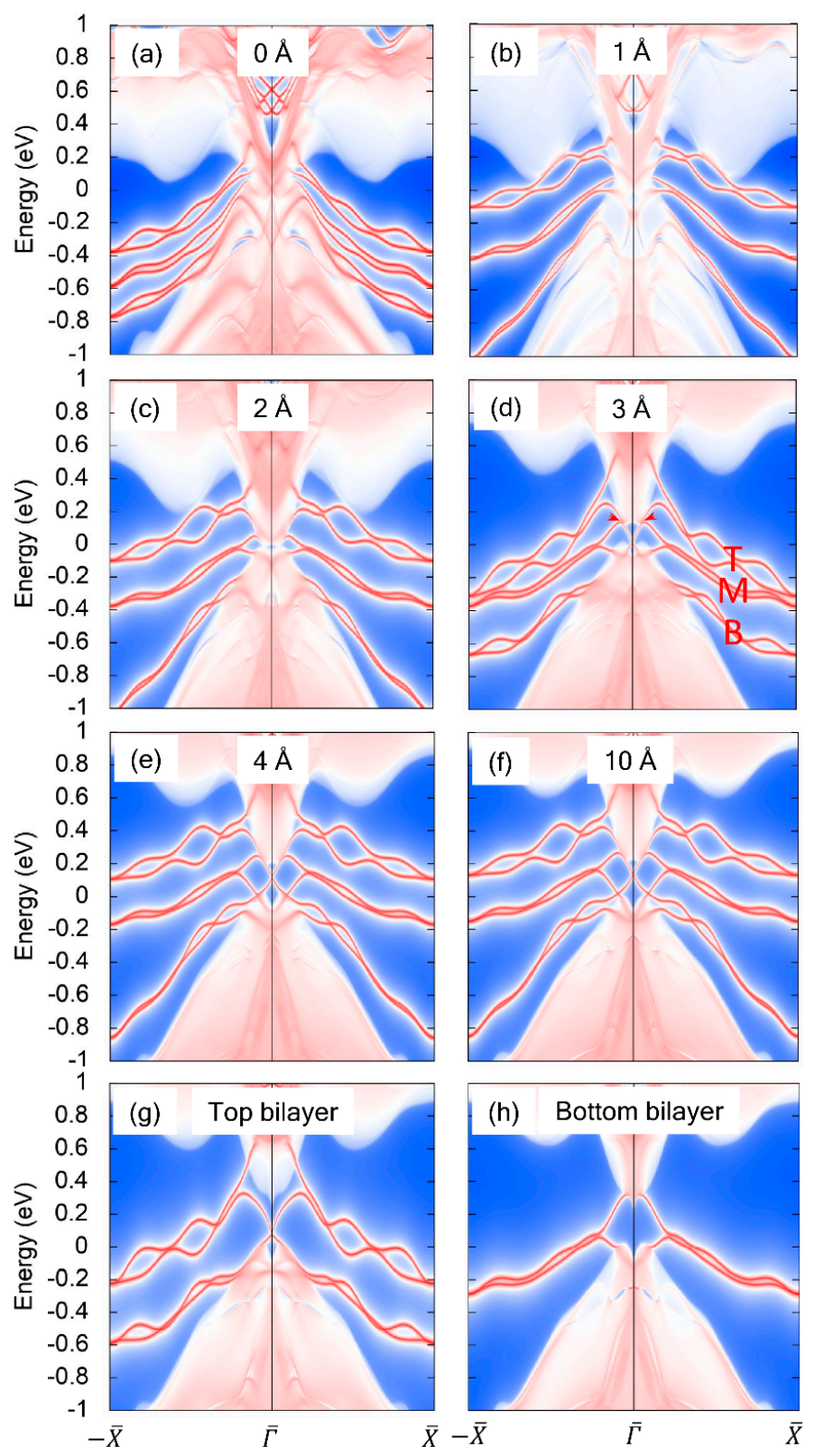

Figure 4. (a-f) are the evolution of edge modes along the zigzag edge of a semi-infinite double-bilayer bismuthene with increasing inter-bilayer spacing as indicated. $(\mathbf{g}, \mathbf{h})$ are the edge modes of individual top bilayer and bottom bilayer of a double-bilayer bismuthene.

The topological nature variation of a double-bilayer bismuthene across the critical spacing can be further understood by its topological edge states. The topological nontriviality of a system can 
be determined by counting the times that its edge modes intersects with its Fermi level between two TRIMs [32]. The intersection times were always even for the systems with an extra inter-bilayer spacing over $2 \AA$ (Figure $4 \mathrm{~d}-\mathrm{f}$ ), regardless of the opening or closing of the anti-crossing gap. This result is in agreement with the fact that the systems with a spacing over $2 \AA$ were topologically trivial, as determined above. We also calculated the edge spectra of the individual top bilayer and bottom bilayer as shown in Figure 4g,h, which helped us to ascribe the edge mode to individual bilayer of a coupled double-bilayer bismuthene. Obviously, the M pair originated from the bottom bilayer and the other two pairs were derived from the top bilayer. The odd times of intersecting between the Fermi level and edge mode both in Figure 4g,h cases shows the nontrivial nature of the individual bismuthene bilayer. When two such individual bilayers were stacked together with large spacing, the spectra of edge mode of an individual bilayer superimposed with each other and made the intersection times even, resulting in a topologically trivial composite system. We noticed the slight difference in the edge state spectra of the individual bilayer and that of the double-bilayer with $10 \AA$ extra spacing. This discrepancy should not be attributed to the interaction between two bilayers, but to the errors introduced in the wannierization process. In reality, the edge atom inclines to go through a reconstruction to minimize energy, and the dispersion of edge modes will change markedly [33]. However, the way in which the topological edge modes connected, as constrained by their topological symmetry, remains unchanged.

\section{Conclusions}

In this paper, we examined theoretically and conceptually the band structure transformation in double-bilayer bismuthene by tuning the inter-bilayer spacing. The double-bilayer at its natural inter-bilayer spacing is a topological insulator. When the spacing increased, the gap decreased and closed at an extra spacing of $d=2 \AA$. Upon further increasing $d$, an inversed gap opened up and the system became a topologically trivial band insulator. The band inversion was realized by reordering the highest valence band and the second lowest conduction band with opposite parity, and this unusual band inversion arose from the $p_{z}$ characteristics of the states involved in the band inversion. There were three pairs of edge bands residing in the bulk gap, and the dispersion of the topologically related one was sensitively dependent on the topological nature of the bulk. As the inter-bilayer spacing was tuned, the dispersion of the edge modes was altered accordingly. Our findings here theoretically demonstrated the transformability of the topological property of double-bilayer bismuthene and the dispersion of its edge modes with inter-bilayer spacing, which may be of valuable reference for the future spintronic application of bismuthene films.

Author Contributions: Conceptualization, X.W.; investigation, H.H.; project administration, X.W.; writingoriginal draft, H.H. and X.W.; Writing - review and editing, Z.S., P.W., W.Z., T.-C.C. and X.W.

Funding: This work received support from the National Natural Science Foundation of China (No. 11204133 for XXW), the Fundamental Research Funds for the Central Universities (No.30917011338 for XXW), the US Department of Energy, Office of Science (Grant DE-FG02-07ER46383 for TCC), the National Natural Science Foundation of China (No. 11504207 for PW), the Shandong Province Natural Science Foundation of China (No. ZR2014AQ006 for PW).

Conflicts of Interest: The authors declare no conflict of interest.

\section{References}

1. Hasan, M.Z.; Kane, C.L. Colloquium: Topological insulators. Rev. Mod. Phys. 2010, 82, 3045. [CrossRef]

2. Niu, Q.; Thouless, D.; Wu, Y.-S. Quantized Hall conductance as a topological invariant. Phys. Rev. B 1985, 31 , 3372-3377. [CrossRef]

3. Roushan, P.; Seo, J.; Parker, C.V.; Hor, Y.S.; Hsieh, D.; Qian, D.; Richardella, A.; Hasan, M.Z.; Cava, R.J.; Yazdani, A. Topological surface states protected from backscattering by chiral spin texture. Nature 2009, 460, 1106-1109. [CrossRef] [PubMed] 
4. Wray, L.A.; Xu, S.-Y.; Xia, Y.; Hsieh, D.; Fedorov, A.V.; San Hor, Y.; Cava, R.J.; Bansil, A.; Lin, H.; Hasan, M.Z. A topological insulator surface under strong Coulomb, magnetic and disorder perturbations. Nat. Phys. 2011, 7, 32-37. [CrossRef]

5. Bernevig, B.A.; Hughes, T.L.; Zhang, S.C. Quantum Spin Hall Effect and Topological Phase Transition in HgTe Quantum Wells. Science 2006, 314, 1757-1761. [CrossRef] [PubMed]

6. Pesin, D.; MacDonald, A.H. Spintronics and pseudospintronics in graphene and topological insulators. Nat. Mat. 2012, 11, 409-416. [CrossRef]

7. Li, S.S.; Ji, W.X.; Li, P.; Hu, S.J.; Cai, L.; Zhang, C.W.; Yan, S.S. Tunability of the Quantum Spin Hall Effect in Bi(110) Films: Effects of Electric Field and Strain Engineering. ACS Appl. Mater. Interfaces 2017, 9, 21515-21523. [CrossRef]

8. Zhang, R.-W.; Zhang, C.-W.; Ji, W.-X.; Li, P.; Wang, P.-J.; Li, S.-S.; Yan, S.-S. Silicon-based chalcogenide: Unexpected quantum spin Hall insulator with sizable band gap. Appl. Phys. Lett. 2016, 109, 182109. [CrossRef]

9. Wang, Z.; Chen, L.; Liu, F. Tuning Topological Edge States of Bi(111) Bilayer Film by Edge Adsorption. Nano Lett. 2014, 14, 2879-2883. [CrossRef]

10. Murakami, S. Quantum spin Hall effect and enhanced magnetic response by spin-orbit coupling. Phys. Rev. Lett. 2006, 97, 236805. [CrossRef]

11. Yaginuma, S.; Nagaoka, K.; Nagao, T.; Bihlmayer, G.; Koroteev, Y.M.; Chulkov, E.V.; Nakayama, T. Electronic Structure of Ultrathin Bismuth Films with $\mathrm{A}_{7}$ and Black-Phosphorus-like Structures. J. Phys. Soc. Jpn. 2008, 77, 014701. [CrossRef]

12. Koroteev, Y.; Bihlmayer, G.; Chulkov, E.; Blügel, S. First-principles investigation of structural and electronic properties of ultrathin Bi films. Phys. Rev. B 2008, 77, 045428. [CrossRef]

13. Hirahara, T.; Bihlmayer, G.; Sakamoto, Y.; Yamada, M.; Miyazaki, H.; Kimura, S.-I.; Blügel, S.; Hasegawa, S. Interfacing 2D and 3D Topological Insulators: Bi(111) Bilayer on $\mathrm{Bi}_{2} \mathrm{Te}_{3}$. Phys. Rev. Lett. 2011, 107, 166801. [CrossRef] [PubMed]

14. Yang, F.; Miao, L.; Wang, Z.; Yao, M.-Y.; Zhu, F.; Song, Y.; Wang, M.-X.; Xu, J.-P.; Fedorov, A.; Sun, Z.; et al. Spatial and Energy Distribution of Topological Edge States in Single Bi(111) Bilayer. Phys. Rev. Lett. 2012, 109, 016801. [CrossRef]

15. Liu, Z.; Liu, C.-X.; Wu, Y.-S.; Duan, W.-H.; Liu, F.; Wu, J. Stable Nontrivial Z 2 Topology in Ultrathin Bi (111) Films: A First-Principles Study. Phys. Rev. Lett. 2011, 107, 136805. [CrossRef] [PubMed]

16. Hirahara, T.; Fukui, N.; Shirasawa, T.; Yamada, M.; Aitani, M.; Miyazaki, H.; Matsunami, M.; Kimura, S.; Takahashi, T.; Hasegawa, S.; et al. Atomic and Electronic Structure of Ultrathin Bi(111) Films Grown on $\mathrm{Bi}_{2} \mathrm{Te}_{3}(111)$ Substrates: Evidence for a Strain-Induced Topological Phase Transition. Phys. Rev. Lett. 2012, 109, 227401. [CrossRef] [PubMed]

17. Chen, L.; Wang, Z.F.; Liu, F. Robustness of two-dimensional topological insulator states in bilayer bismuth against strain and electrical field. Phys. Rev. B 2013,87, 235420. [CrossRef]

18. Zhou, J.-J.; Feng, W.; Liu, C.-C.; Guan, S.; Yao, Y. Large-gap quantum spin Hall insulator in single layer bismuth monobromide $\mathrm{Bi}_{4} \mathrm{Br}_{4}$. Nano Lett. 2014, 14, 4767-4771. [CrossRef] [PubMed]

19. Li, X.; Liu, H.; Jiang, H.; Wang, F.; Feng, J. Edge engineering of a topological Bi(111) bilayer. Phys. Rev. B 2014, 90, 165412. [CrossRef]

20. Aktürk, E.; Aktürk, O.Ü.; Ciraci, S. Single and bilayer bismuthene: Stability at high temperature andmechanical and electronic properties. Phys. Rev. B 2016, 94, 014115. [CrossRef]

21. Huang, Z.-Q.; Chuang, F.-C.; Hsu, C.-H.; Liu, Y.-T.; Chang, H.-R.; Lin, H.; Bansil, A. Nontrivial topological electronic structures in a single $\mathrm{Bi}(111)$ bilayer on different substrates: A first-principles study. Phys. Rev. B 2013, 88, 165301. [CrossRef]

22. Gonze, X. A brief introduction to the ABINIT software package. Z. Kristallogr. Cryst. Mater. 2005, 220, 558-562. [CrossRef]

23. Gonze, X.; Amadon, B.; Anglade, P.-M.; Beuken, J.-M.; Bottin, F.; Boulanger, P.; Bruneval, F.; Caliste, D.; Caracas, R.; Côté, M. ABINIT: First-principles approach to material and nanosystem properties. Comput. Phys. Comm. 2009, 180, 2582-2615. [CrossRef]

24. Hartwigsen, C.; Gøedecker, S.; Hutter, J. Relativistic separable dual-space Gaussian pseudopotentials from $\mathrm{H}$ to Rn. Phys. Rev. B 1998, 58, 3641-3661. [CrossRef] 
25. Monkhorst, H.J.; Pack, J.D. Special points for Brillouin-zone integrations. Phys. Rev. B 1976, 13, 5188-5192. [CrossRef]

26. Wu, Q.; Zhang, S.; Song, H.-F.; Troyer, M.; Soluyanov, A.A. WannierTools: An open-source software package for novel topological materials. Comput. Phys. Commun. 2018, 224, 405-416. [CrossRef]

27. Grimme, S. Semiempirical GGA-type density functional constructed with a long-range dispersion correction. J. Comput. Chem. 2006, 27, 1787-1799. [CrossRef]

28. Slater, J.C.; Koster, G.F. Simplified LCAO method for the periodic potential problem. Phys. Rev. 1954, 94, 1498-1524. [CrossRef]

29. Liu, C.-X.; Zhang, H.; Yan, B.; Qi, X.-L.; Frauenheim, T.; Dai, X.; Fang, Z.; Zhang, S.-C. Oscillatory crossover from two-dimensional to three-dimensional topological insulators. Phys. Rev. B 2010, 81, 041307(R). [CrossRef]

30. Zhang, Y.; He, K.; Chang, C.-Z.; Song, C.-L.; Wang, L.-L.; Chen, X.; Jia, J.-F.; Fang, Z.; Dai, X.; Shan, W.-Y.; et al. Crossover of three-dimensional topological insulator of $\mathrm{Bi}_{2} \mathrm{Se}_{3}$ to the two-dimensional limit. Nat. Phys. 2010, 6, 584-588. [CrossRef]

31. Yazyev, O.V.; Moore, J.E.; Louie, S.G. Spin Polarization and Transport of Surface States in the Topological Insulators $\mathrm{Bi}_{2} \mathrm{Se}_{3}$ and $\mathrm{Bi}_{2} \mathrm{Te}_{3}$ from First Principles. Phys. Rev. Lett. 2010, 105, 266806-266809. [CrossRef] [PubMed]

32. Fu, L.; Kane, C.L. Topological insulators with inversion symmetry. Phys. Rev. B 2007, 76, 045302. [CrossRef]

33. Peng, L.; Xian, J.-J.; Tang, P.; Rubio, A.; Zhang, S.-C.; Zhang, W.; Fu, Y.-S. Visualizing topological edge states of single and double bilayer Bi supported on multibilayer Bi(111) films. Phys. Rev. B 2018, 98, 245108. [CrossRef]

(C) 2019 by the authors. Licensee MDPI, Basel, Switzerland. This article is an open access article distributed under the terms and conditions of the Creative Commons Attribution (CC BY) license (http://creativecommons.org/licenses/by/4.0/). 